\title{
Intent For Prophylaxis
}

National Cancer Institute

\section{Source}

National Cancer Institute. Intent For Prophylaxis. NCI Thesaurus. Code C54404.

The use of a particular medical device, medication or treatment to help reduce the risk of contracting or developing a disease or condition. 